\title{
Performance Evaluation of IoT Messaging Protocol Implementation for E-Health Systems
}

\author{
M. Zorkany ${ }^{1}$ \\ National Telecommunication \\ Institute (NTI) \\ Cairo, Egypt
}

\author{
K.Fahmy ${ }^{2}$ \\ Department of Electrical Engineering \\ Al-Azhar University, Nasr City \\ Cairo, Egypt
}

\author{
Ahmed Yahya ${ }^{3}$ \\ Department of Electrical Engineering \\ Al-Azhar University \\ Cairo, Egypt
}

\begin{abstract}
Now-a-days, e-health and healthcare applications in the internet of things are growing rapidly. These applications are starting from remote monitoring of patient's parameters in home to monitoring patients during his life activities at work, transportation, etc. So we can monitor patients at any place outside of hospitals and clinical settings. By using this technology, we can save lives and reduce the number of emergency visits to hospitals. In contemporary time, there are great progress and opportunities for the internet of things (IoT) related E-health systems. Most IoT e-health platforms consist of three main parts; client nodes (patient or doctor), IoT server and IoT communication messaging protocol. One of E-health systems design over IoT challenge is choosing the most suitable IoT messaging protocol for E-health applications. In this paper, IoT remote patient and e-Health monitoring system was designed for monitoring physiological medical signals of patients based on most two famous IoT messaging protocols, MQTT and CoAP. These medical signals can be include parameters like heart rate signals, electro-cardio graph (ECG), patient temperature, blood pressure, etc. This practical comparison between CoAP and MQTT is to choose most suitable for e-health systems. The proposed approach was evaluated based on most significant protocol parameters like capability, efficiency, communication method and message delay. Practical and simulation results show the performance of the proposed E-health systems over IoT for different network infrastructure with different losses percentages.
\end{abstract}

Keywords-E-health; IoT; IoT protocol; CoAP; MQTT and remote patient monitoring

\section{INTRODUCTION}

IoT (Internet of Things) is an extension of the current internet by connecting millions of devices (things) together nevertheless the location of each thing. So IoT is a network between devices or things through any internet connection method (wire, wireless, WiFi, GPRS, 3G, etc). IoT applications grow in many applications like transportations, medical, shopping, smart home, smart cities etc. To build internet of thing platform for any application we need internet connections between clients and communication protocol to manage these connections through IoT server.

Nowadays the market of IoT in medical applications espial for e-health is growing rapidly ranging from medical smart sensors and remote patient monitoring to remote traceability and diagnosis of patients. The main objective of e-health over IoT is improving the health of patients through better disease management and self-help facilities.
IoT e-health systems can be classified as an Internet of Medical Things (IoMT). IoMT is a medical sensors and applications connected through IoT network. Also IoMT can be use Web Services, to captured patient data and analyzed it. The general architecture of e-health system [1] shown in Fig. 1 which consists of three main phases: Phase 1 monitoring the patient to acquire data from medical sensors, then this data is gathered and transferred through the internet to the application server or clients for data analysis, monitoring and investigation in third phase. Also some of data processing can be accomplished at patient side and send the results to doctor's side directly.

IoT platform has three main parts: clients, IoT server and IoT message protocols. As shown in Fig. 2 E-health monitoring system architecture [2] divided into three layers: sensing layer. It is a collection of medical sensors. server layers It links between the sensing layer "patient" and receiving data layer "hospital". Receiving data layer is the layer through which patient data are received. In this article, rely on the server layer to choose protocol most suitable for the electronic health system.

The messaging protocol is considered the main element in the Internet of things. These protocols play a big role to enable IoT all over life. One of E-health systems design over IoT is to choose the type of IoT messaging protocol. In the Internet of things the main factor is the incorporation of many technologies, due to the fast growth of the Internet of things information sharing has been sophisticated across systems where the internet is converted into a comprehensive future [3]. Internet of things has a great ability to grow and the main engine of this growth is the protocol [4]. So using dedicated protocols to the internet of things will increase the efficiency and ease of data transfer certified on the environment used. In recent years, health care applications have received a lot of attention, making researchers discuss the whole structure of the healthcare system through IoT and applicability [5].

The electronic health system has maintenance medical devices, way of managing hospitals and Patients were allowed arrival to medical care all hospitals in the world. Many hospitals have implemented an electronic health system to solve the problems of shortage of doctors, reduce costs for patients and increase efficiency [6]. The electronic health system aims to progress the health aspect where it monitors the organs of the human body and its vital functions, which helps to decrease the mortality rate. In the future, health care 
applications across IoT will have a great effectiveness the state economy [7].

At this time, there is a lot of messaging protocols for IoT, some of which are distinguished according to a need for them. IoT protocol is divided into: TCP/IP protocol Such as: MQTT, SMQTT, AMQP and UDP protocol Such as: CoAP, SNMP, SSI. [8] Internet of things has major importance in electronic health so it was thought to this part of the application for the Internet of things to develop a Special Protocol for E-health to increase efficiency and care for patient cases in this paper, each protocol will be expounded separately to choose the most suitable protocol for health care system. There shall be a practical Comparison amongst the most renowned protocols it "MQTT, CoAP". This comparison will be based on the Special process components of each protocol.

In this research, e-health IoT platform will be implemented depending on most two famous IoT protocols MQTT (ex. TCP) and CoAP (ex. UDP). This practical comparison between CoAP and MQTT is to choose the protocol most suitable for e-health systems. The proposed approach was evaluated based on most important internet parameters like capability, efficiency and message delay. Practical results show a performance of E-health systems over it in different network infrastructure with different losses percentages. This comparison was presented to illustrate the integrity and health information towards the protocol most suitable for electronic health.

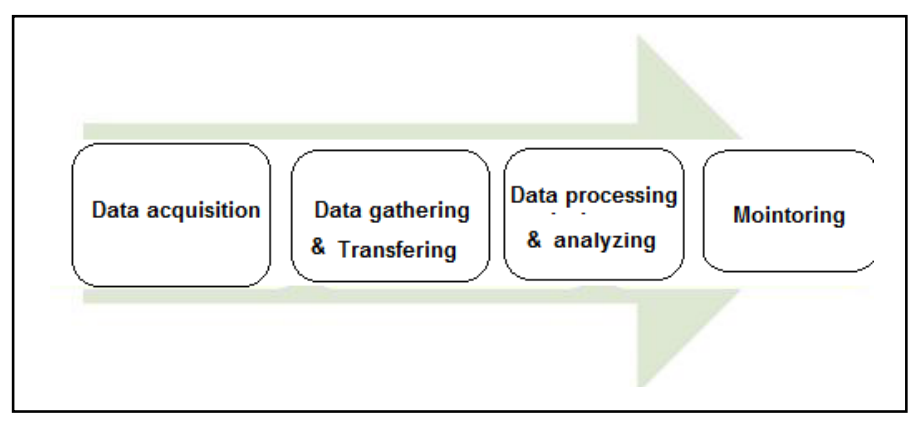

Fig. 1. General Architecture of e-Health System.

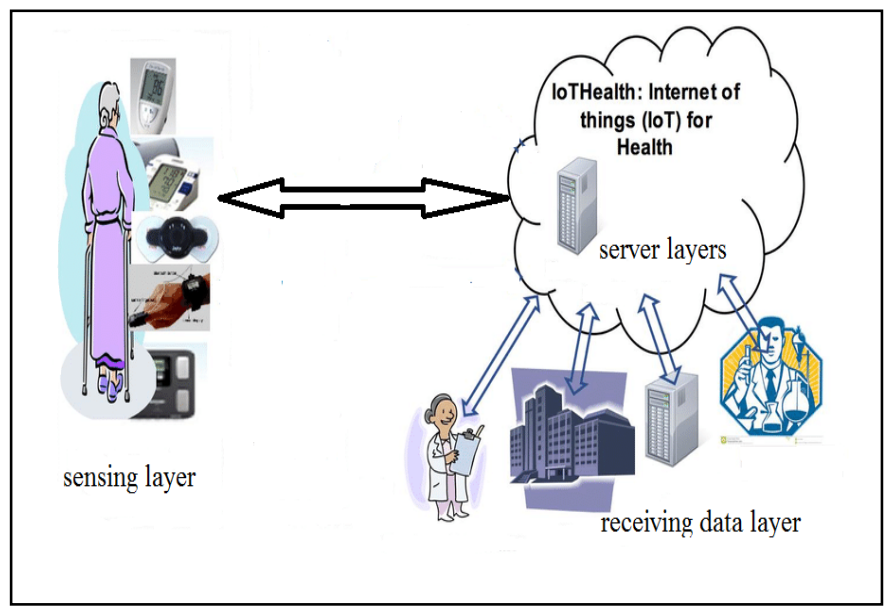

Fig. 2. e-Health Monitoring System Architecture.
This paper is regular as follows: Section 2 presents related of work IoT protocol. Section 3 discusses the famous IoT protocols. Then, Section 4 Proposed criteria for selecting the most protocol appropriate for the E-health application, in Section 5 set up scenario and practical results, the concludes of this research will be discussed in Section 6.

\section{RELATED WORK}

Most e-health over IoT studies and researches in remote monitoring of patients although quite complete but are used for individual problems like as ECG or heart rate monitoring. Some studies developed for special features as develop prototypes for electrocardiography integrated with smart phone [9]. In recent years, many researches applied internet of things in many e-health applications. To develop these applications over IoT they used standard IoT protocols. For example, R.N. Kirtana, Y.V. Lokeswari in Paper [10] developed Heart Rate Variability (HRV) system to remote heart rate monitoring over IoT using MQTT protocol. But this system developed to monitoring one medical signal (heart rate) only. Antoine Jamin et al. in paper [11] presented an inhome aggregation IoT platform to monitoring more than one medical parameters (physiological and thermometer parameters). They used standard IoT protocol to implement their platform. So they send one message per parameter because MQTT is single topic protocol. In this case if patient has more than one emergency parameter in the same time, they must send one parameter in one MQTT message, and then transmit next parameter in other MQTT message. Which cause a delay in some message and occupy more internet bandwidth.

Kaleem Ullah et al. in paper [12] proposed framework for e-Health which used smart phone sensors with body sensors to monitor patient health. They used different protocol as HTTP and HTTPS. But they didn't use most famous IoT protocols like MQTT or CoAP. M. Hussein et al. developed IoT protocol based on proposed multi-topic IoT protocol which can send multi parameters in one message to overcome delay of multi messages and save the bandwidth [13]. Also, several researchers shared their efforts to develop the health care framework across IoT for example: P. Thota and Y. Kim compared the famous IoT Protocols MQTT and CoAP based on Raspberry-Pi and a temperature sensor [14]. The Pros and Cons of each of them in terms of the power consumption and data, flows from one node to multiple nodes, safely. CoAP more functional in terms of the energy, while the MQTT more suitable when data flows from a client to multiple nodes, but this consists of only two factors and neglect other factors.

K. Natarajan et al. used Raspberry Pi in E-health over IoT to collect patient data through the sensors, store it and send to the doctor [15]. Raspberry $\mathrm{Pi}$ is capable to collect diverse information from the patient through sensors. Mentioned how the data were collected but he did not talk about anything concerning the method of sending data over the protocol used in IoT. A. Al-Fuqaha et al. provide an overview about IoT, basic elements, tools and techniques needed from where communication technologies used in IoT like NFC, Z-WAVE, LTE-A, Bluetooth LE and RFIC [16]. In addition to the most renowned IoT messaging protocols as MQTT, CoAP XMPP, 
AMQP. Description of different criteria for each protocol and explained the using of each of them in detail. Explained how to collect information by previous communication technologies and send off them through those protocols to achieve the goal of IoT. This paper didn't mention the challenges facing the protocols and the differences application between all these protocols. Also, they didn't run the protocols mentioned in real applications of the IoT, but mentioned in a theoretical way only. They didn't address the search of the best protocols from where energy and efficiency and the capacity to send data. It was necessary to mention the best protocol for each part of the different IoT.

T. Takpor and A. Atayero talked about the dangerous matter of integrating E-Health and educational institutions across IoT to effectively monitor students' health [17]. Because health issues affect students' academic performance, so exhibited a technique 'RFID' Radio Frequency Identification, and use it to know the medical data of students such as "blood".

There a lot of work on different directions for the achievement of that project from sensors used and accuracy and how to compile data and keep it and how to transmit data Through IoT protocols. In this article Show the effect of IoT on improving electronic health, clarifying the communication models and protocols used in IoT and health care implementation. Today can be applied mobile devices in monitoring patients and the elderly and identifying their health status. The problem is how to join these devices to the internet world while preserving the safety of information. It was necessary to have a look at a collection of IoT messaging protocols used to solve the problems and to apply the application to transmit data [18].

From the above survey, we find that most researches didn't talk about the best protocols that ability to use in E-health applications. In our research, we will do a comparison between most IoT famous protocols to select the most appropriate protocol for the healthcare system. In the next section, clarification the most renowned IoT protocol will be discussed.

\section{IoT MESSAGE PROTOCOL}

IoT message protocols can be divided into TCP/IP and UDP protocol. The most popular TCP protocol is "MQTT" and one of the most renowned UDP protocols is "CoAP".

\section{A. MQTT "Message Queue Telemetry Transport"}

It is a publish-subscribe messaging TCP/IP protocol. The messaging style "publish-subscribe" need to IoT server "broker". The publish send the message to the broker, the broker is accountable for classified messages to interested clients relying on the topic of a message as illustrated in the Fig. 3. It has many advantages as short massage, minimized data packets, faster response and throughput 'Speed', low power usage and lower bandwidth, Other advantages built into the MQTT protocol are retained messages and multiple subscriptions 'multiplexed' over one connection. many-tomany communication protocol for crossing messages between multiple clients through the broker.

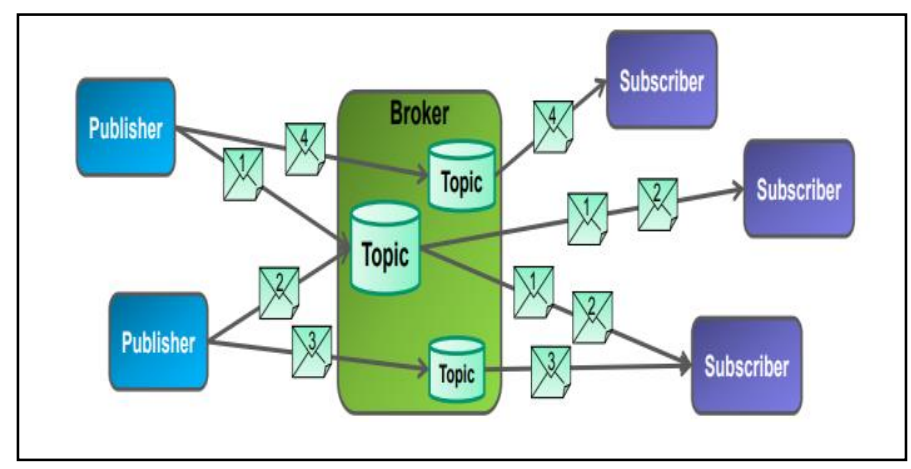

Fig. 3. General MQTT Architecture.

MQTT has featured it supports Quality of Service (QoS). The Quality of Service (QoS) is a method to secure the submission of the message between publisher and subscriber. There are three standards of Qualiy of Service (QoS), the standard of Quality of Service (QoS) is determined as in paper [19] within the scope of the research, it was found that there were some letters that showed official models of MQTT as well as how to pass messages and analyze them a constant analysis based on indications in paper [20]. MQTT protocol has the ability to save the order between the messages by adding the mark of the request and SEO which determines whether you want to keep the order between the messages or not.

\section{B. CoAP "Constrained Application Protocol"}

It is a "Request/ Response" protocol, it is used as an alternative to http in hardware-constrained devices to be simple to use with these devices so it is used in IoT. The CoAP provides four different types of messages:

- The message type defines four different method:

CON Message: refers to the "Confirmable" request. When a source node transmits a $\mathrm{CON}$ request, the recipient has to respond with ACK message.

NON Message: refers to "Non-Confirmable" request that when a source node transmits a non-request, the recipient is not required to respond back.

ACK Message: refer to "Acknowledgement" messages which are sent back as a response to a $\mathrm{CON}$ message. If processing successful, the recipient of the CON message should reply back with an ACK message. The ACK message can also contain the result of the processing time.

RST Message: refer to "Reset" message this type of messages is sent back when the future of a message encounters an error, does not understand the message or is no longer interested in the message sender.

- The message codes define one method: empty message.

- Request codes define four different methods:

(GET) This will retrieve the information from a specific resource specified by requested URI (POST) display information to be processed for a specific resource. The output result depends on the targeting resource, usually, results in the target resource that is created or updated. (PUT) requests that 
resource particular by the URI be created with the carried information representation; (DELET) demand that the identified resource can be deleted.

- Response codes define two different methods :

Success message Contains four messages: CONTENT message response for GET message, CONTIUE message response for POST message, CREATED message response for PUT message, DELETED message response DELETED message.

Client error message Contains different message: such as bad request, not found, method not allow.

General CoAP architecture is exhibit in Fig. 4.

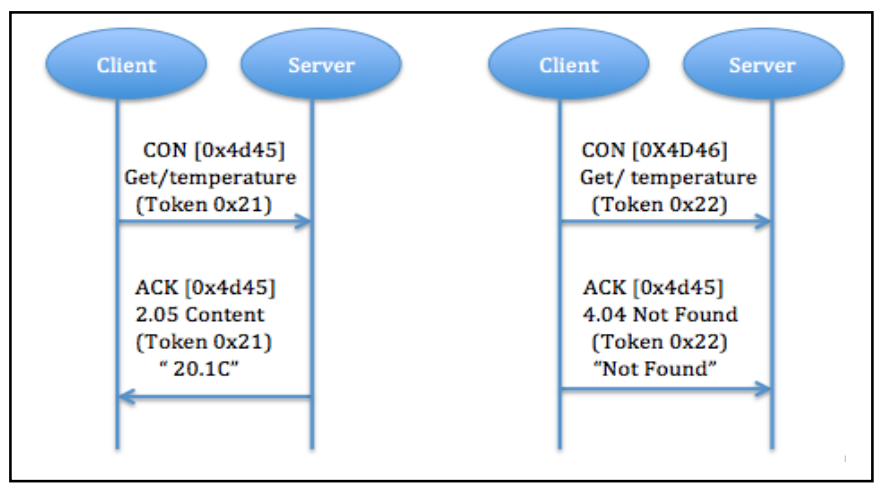

Fig. 4. General CoAP Architecture.

\section{PROPOSED PERFORMANCE EVAlUATION CRITERIA FOR E_HEALTH OVER IOT}

The proposed e-health system is An automatic wireless health monitoring system which can be used to measure patient's parameters (like body temperature and heartbeat, etc.) by using embedded system technology with internet of things. As shown in Fig. 5, The architecture of proposed system divides into five parts: Patient (sensor) node, IoT communication protocol, IoT server, application server and application node.

The patient node unit is medical sensors, internet access, controller and power and surrounded circuits. Medical sensors used to acquire medical parameters from patients. Selection of medical sensors depend on two issues decisions: which required sensing parameters need monitored, and which sampling frequency needed to send theses parameters to doctors/Hospital or sending these parameters only when required or at abnormal conditions. In order to the patient node achieve the objectives of the system; the scope of the node is summarized as follow:

Microcontroller system controls the operation of gathering the data from sensors and preparing it to be sent. It is integrated with some extra component to increase the functionality and execute at high frequency to improve the performance of the system so that the system is more reliable and efficient. Internet access like Wi-Fi or Bluetooth or GSM/GPRS/3G module used to transmit and receive data wirelessly in a long distance so the system is portable and easy to be operated.

\section{A. IoT Protocol Architecture}

In order that achieves better healthcare in the Internet of things, need to transfer data of patients accurately and effectively to remote servers. This paper proposed method to select the most convenient messaging protocol for the healthcare system. The architecture of the protocol system appropriate for electronic health depends on three layers, Client receiving data, Gateway / Translation Device "Protocols", Sensor data collection "sensing layer" as described in Fig. 6.

The sensing layer consists of many medical sensors such as pressure measurement, temperature, heartbeat, ECG, blood glucose level and breathing rate. Each sensor measures its own patient data and sends it to the doctor through the server layer, in this class, the data enable be collected and rerouted.

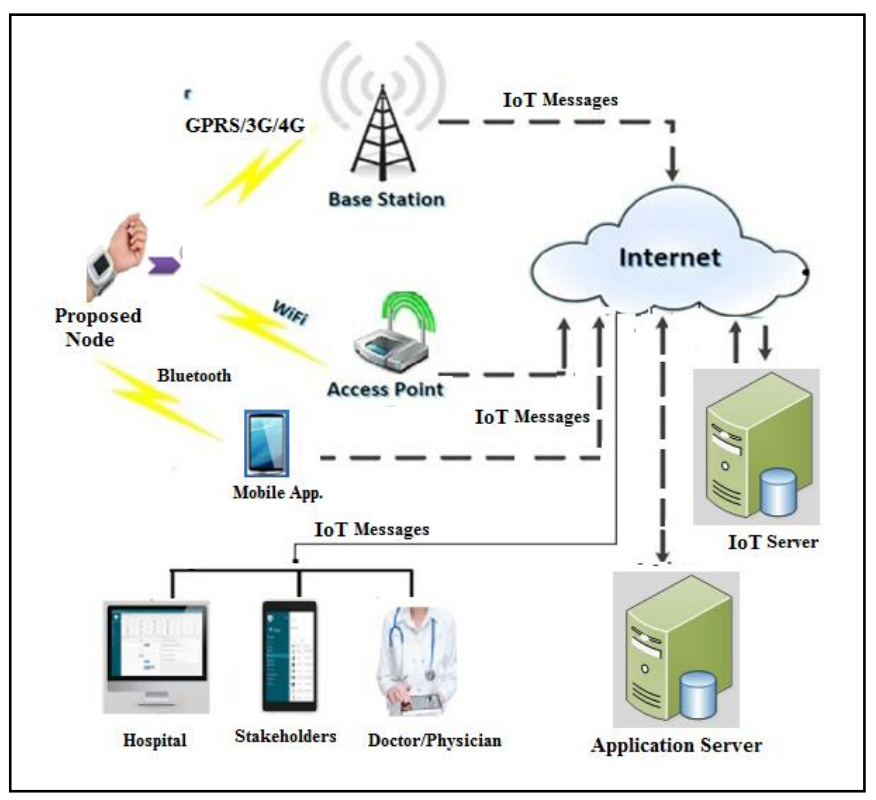

Fig. 5. Proposed IoT Patient Monitoring System.

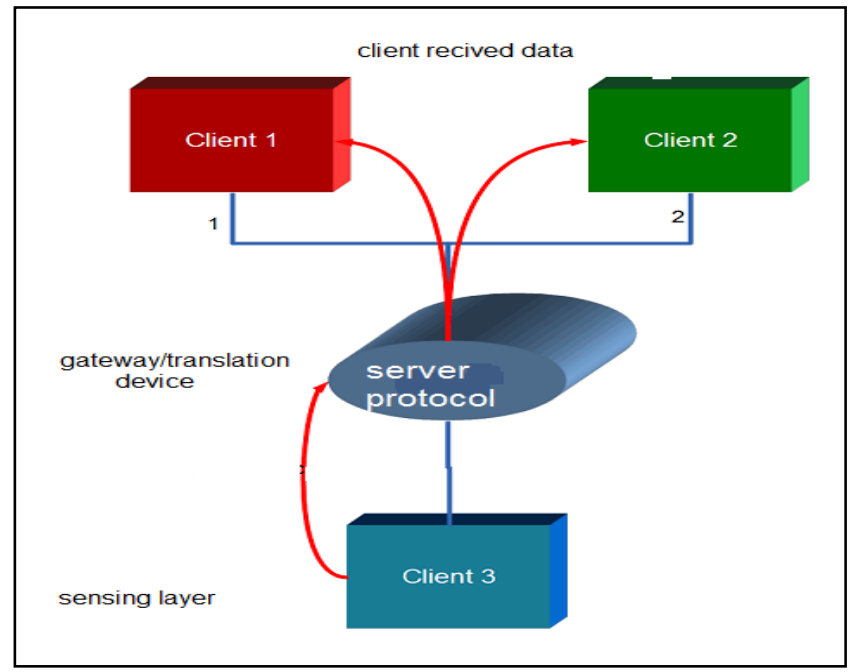

Fig. 6. The Proposed Methodology. 


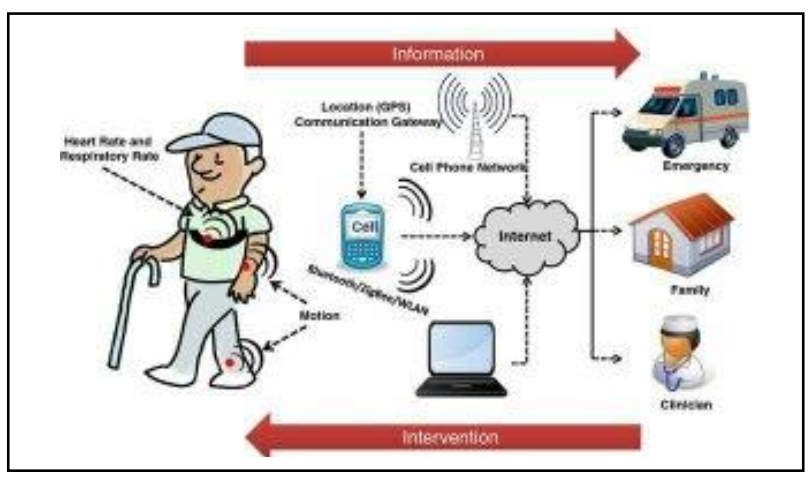

Fig. 7. Remote Health Monitoring.

In the server layer the focus is on communication protocols that authorize the reciprocation of information between devices, where the Internet of things is entered in many fields, whether industrial or health, where information exchange occurs between devices via cloud systems, the link in that is many communication protocols Which allow devices converse to each other and with the connection of millions of devices on the Internet, need for IoT protocols was very important such as MQTT, CoAP looking for whichever is more compatible for electronic health.

In the receiving data layer the information is received by the doctor that was sent in the sensing layer where the information is processed and stored continuously, occur a quick reaction to the emergency and other treatment by the doctor. Fig. 7 shows these three stages.

The first phase "The sensing layer" shows the patient's specific sensors and how to install it and measure the data. the second phase "server layer " describes how to transfer that patient-specific data that has been measured through the medical sensors via the IoT protocols. The third phase "receiving data layer" is the special phase in the hospital where the doctor receives the data of the patient was sent in the previous phase through special applications. Application "client" for each protocol to be the window to transmit data to patients Like MQTT FX data is then transferred to the public network to select most protocol convenient for transfer data. The researchers have made protocols IoT 'internet of things' to suit data transfer, enhance efficiency, safety, and energy consumption, as the devices used in electronic health often operate on batteries, the protocol should be suitable for energy saving and easy to use for the customer.

\section{B. Metrics of the Proposed Method}

The proposed comparison in this article based on three aspects metrics as follows:

1) IoT Success Message "ISM": With this metric, the speed of sending messages is evaluated, this is because the messages that arrive directly are quicker than the messages that need to retransmitting due to loss in the network. This measure is the rate of the total number of messages sent from client to server without the occurrence of retransmitting it, and the gross number of messages sent whether it has to resend or not. pronounced in the equation. all message sent $\mathrm{M}(\mathrm{s})$ consists of:
The successful message $\mathrm{M}$ (succ) from the first time and other message happen to be retransmitted $\mathrm{M}(\mathrm{re})$.

$\mathrm{M}(\mathrm{s})=\mathrm{M}($ succ $)+\mathrm{M}(\mathrm{re})$
$\mathrm{ISM}=\frac{\text { No.of successful messages }}{\text { No.of retransmit and successful message }}$

2) IoT Average Byte "IAB": With this metric, an medium number of bytes used in the message evaluated, because all protocol has its own format regardless of the tenor of the messages. This measurement is the rate of an average number of bytes used in messages sent from the client to the server without retransmit, and the medium number of bytes used in sent messages, whether resend it or not.

$\mathrm{IAB}=\frac{\text { average byte of successful messages }}{\text { average byte of retransmit and successful message }}$

Pronounced in the equation. Packet contains a collection of byte it is a byte of payload $\mathrm{B}(\mathrm{p})$ and byte of 'fixed, variable' header $\mathrm{B}(\mathrm{h})$ specific protocol.

$\mathrm{B}=\mathrm{B}(\mathrm{p})+\mathrm{B}(\mathrm{h})$

3) IoT Delay message "IDM": Message delay is evaluated in this metric, this is ratio of delay of messages sent from the client to the server without retransmission, and delay messages sent, whether resend it or not. This is because most important in E-health is the speed and size of the sending the message and the energy consumption add to the messages lost. Pronounced in the equation.

Delay consists of transmission delay $\mathrm{D}(\mathrm{t})$, signal processing delay $\mathrm{D}(\mathrm{s})$ and queueing delay $\mathrm{D}(\mathrm{q})$.

$\mathrm{D}=\mathrm{D}(\mathrm{t})+\mathrm{D}(\mathrm{s})+\mathrm{D}(\mathrm{q})$

$\mathrm{IBM}=\frac{\text { delay of successful messages }}{\text { delay of retransmit and successful message }}$

As the speed of access to patient information to the doctor to follow the importance of medical, as well as the number of lost messages are a major risk in the diagnosis, the smaller the volume of messages sent the faster the access. Fig. 8 shows a comparison between most prominent IoT protocols MQTT, CoAP. The Patient-specific data "client1" will send to the specialist doctor "client2" through the server of the protocol used.

It is distinguished by the MQTT it supports (QoS). The Quality of Service (QoS) is to secure the transmission of the message between publisher and the future exist three-phases of Quality of Service (QoS), the publisher determine the level of the service.

QoS level 0: message sent from publisher to intermediary and no acknowledgment is sent to a publisher. This part has not been reviewed because it was working on a local network.

QoS level 1: a message sent by the publisher to the intermediary and then the mediator sends an acknowledgment to the publisher again until the publisher ensures that do not miss the message, this means that transmission is guaranteed. 


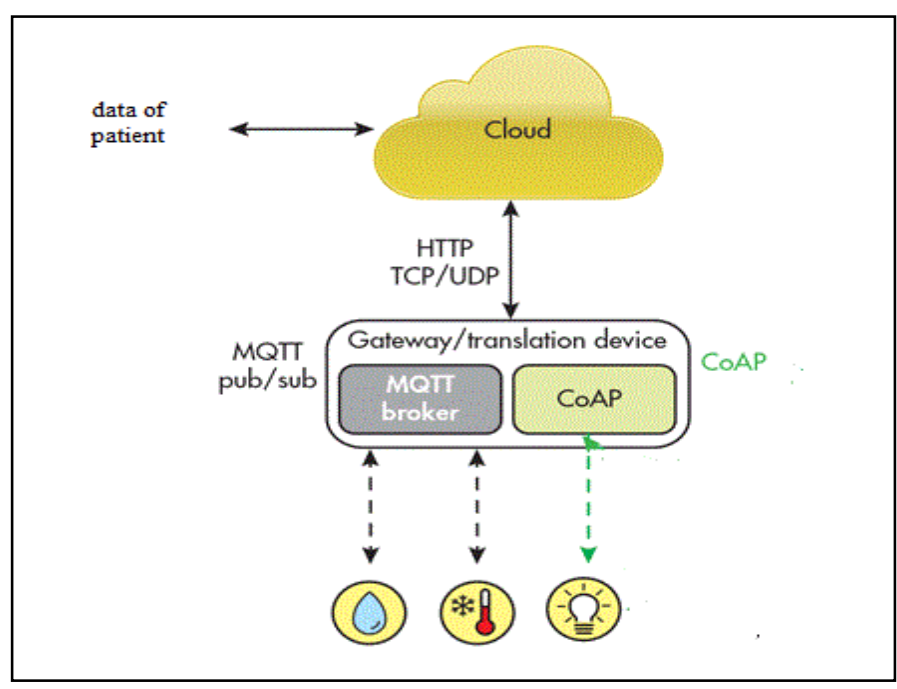

Fig. 8. The Actual Implementation.

QoS level 2: The highest level of service, where there is a chain of four messages between publisher and mediator to confirm sending and confirm receipt.

\section{IMPLEMENTATION OF IOT PROTOCOL AND SET UP SCENARIO}

A typical comparison between CoAP and MQTT and evaluated for appropriate selection, each client has a protocol and a private server.

MQTT implementation: have "MQTT FX" as a client and "MOSQUITO" server. MQTT FX" CLIENT through which the message sent from publisher "patient" and received it by subscriber "Doctor"; subscriber and publisher related to the server with special IP. MOSQUITO" SERVER mosquito is an MQTT broker used for handling data between publisher and subscriber. Receives data from publisher and sends it to the subscriber certified the subject of the message.

CoAP implementation: have "COPPER" client and "LIBCoAP "server. "COPPER" CLIENT through which the message sent from the patient to his doctor connects to the server using the IP of the server. CoAP client use 4 orders (GET) that retrieves information from a specific resource that is particular by the desired URI. (POST) provides the information to be processed to a private resource. The output result depends on target resource, usually, results in the base resource being created or updated. (PUT) requests to create the resource that is particular by the URI or updated with the carried information representation. (DELETE) requests that identified resource be deleted. "LIBCoAP" SERVER it is a special server for CoAP protocol, where data is sent from the patient to server using a command "put" and the doctor takes the patient's data from a server using a command "get".

To simulate the loss of network to show the performance of the proposed e-health systems over IoT at different network infrastructure with different losses percentages, some assisting programs were used as WANEM and WIRE SHARK.
WANEM is an extensive network emulator, designed to provide a real network experience. Which can be used to simulate WAN properties such as network delay, packet damage, packet loss, disconnecting, jitter, reorder of the package, etc. WANEM through which we can change the loss so as not to result in ideal case the WANEM must mediate Client and broker by routing table.

WIRE SHARK supports large number of the protocol. It analyzes the data sent and received through the protocol. Through it know: Public packets and subscribe, Timespan, Average packet per second, Average packet per size, average byte per second. From here we can calculate: IoT Success Message "ISM", IoT Average Byte "IAB" and IoT Delay message "IDM".

The scenario through which we have chosen the appropriate protocol for electronic health. It appears in Fig. 9.

The first scenario was executed using an MQTT protocol, where we have sent the patient's data from the client "MQTT FX" to the attending physician "doctor" through MOSQUITO server. Using WIRESHARK We can tracing the MQTT messages to know which of these messages received from the first time and which of them need re-sent. Through Wireshark, we were Managed these MQTT message to calculate the average byte used in sent messages and the time need to transmitting. All previous measurements in the ideal case, We have varied the loss ratio by WANEM server and re-do the practical simulations and re-measure an calculate the average byte and time to transmitting messages.

The second scenario was implemented using the CoAP protocol. We sent the patient's data from the CoAP client "CoPPER" to the doctor by the LIBCoAP server passing with WIRESHARK. By tracing the sent data to show which messages received at destenation "doctor". Also via WIRESHARK analysis we can calculate the average byte used to send messages and the user time to transmitte. all the previous measurements in the ideal case, then we have change the loss ratio using WANEM simulator and re-measure all the previous parameters to study the performance of the protocol in different network conditions.

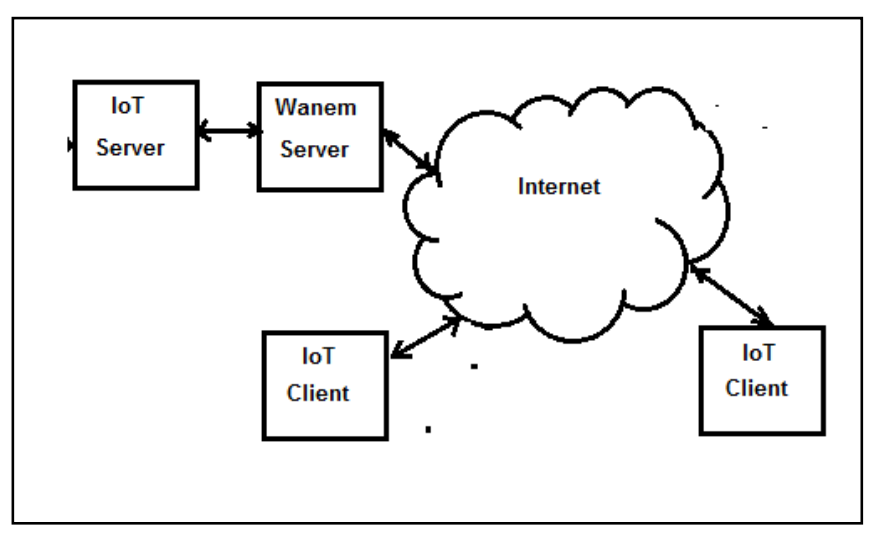

Fig. 9. Set up Scenario. 


\section{PRACTICAL AND SimUlation RESUlts}

The main objective of the practical results and simulation is to clarify which protocols "MQTT, CoAP" are more suitable for electronic health where the results were evaluated by three factors. "ISM, IAB, IBM". From the practical and simulation results:

1) As shown in Fig. 10, the percentage of successful messages in case of the MQTT protocol greater than CoAP protocol, MQTT has a quality of service so the result is better in case of increasing the loss.

The CoAP protocol is special case of UDP protocol. And cannot return lost messages and does not contain QoS, if there is a loss of patient data, the data cannot be retrieved and therefore cannot be used CoAP protocol in medical cases because the doctor need all the patients data without missing anything so that he can Correct diagnosis and ensure improved health care.

2) As shown in Fig. 11, the rate of average byte in case CoAP greater than MQTT in the case of increasing the loss precentage. Despite what appeared in the first curve of the defect of the Coap protocol but it appears the second drawback is the large increase in the number of bytes used to send the message. Increasing the size leads to a large load on the network causing some messages to be lost. In spite of QoS 2 in case MQTT needs to send a series of messages to confirm receipt of message it needs a number of bytes less than the CoAP, does not mean increasing the size of messages in QoS 2 for their size in QoS 1 use QoS 1, However, we need to use QoS 2 in medical cases to ensure patient data is correct. There are also features in MQTT protocol it moves between Quality of Services automatically. You choose QoS 2 and the network does not allow to use because of a problem in it Selects QoS 1 so the network service is improved and then the choice is back QoS 2 again.

3) As shown in Fig. 12, The ratio delay of successful message in case CoAP greater than MQTT in the case of increasing the loss percentage.

Here is talk about the delay in time, it turns out that the delay in QoS2 is greater than QoS1 and CoAP because it sends a series of messages to ensure the arrival of the message carrying the patient's data. From here we can say that the applications that need to be accuracy in sending the message do not consider the time delay being used QoS 2, but the Applications that care more about time are used QoS 1. In both cases, MQTT is more appropriate than CoAP.

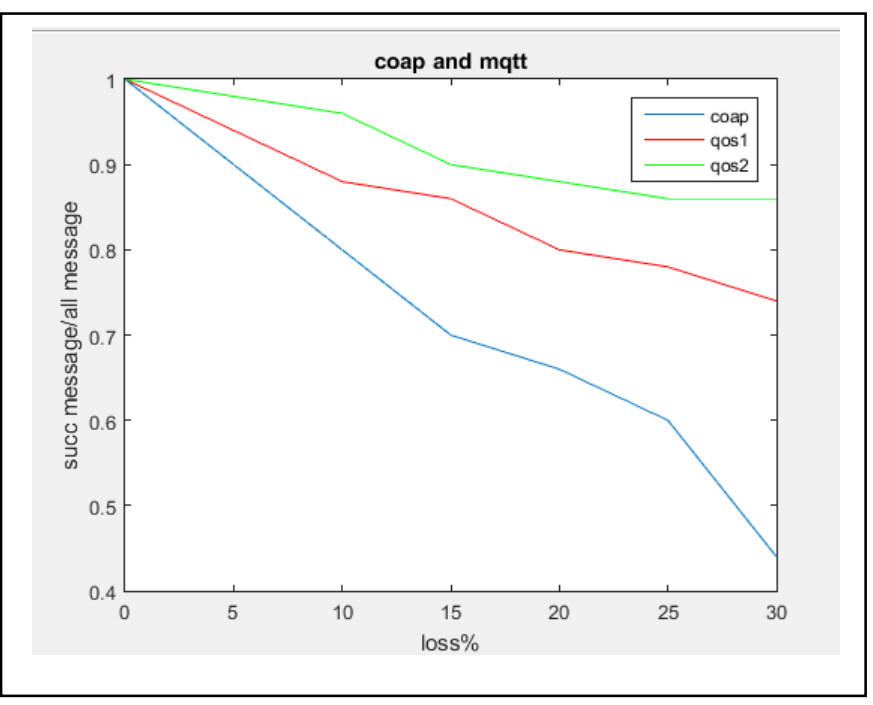

Fig. 10. IoT Success Message "ISM".

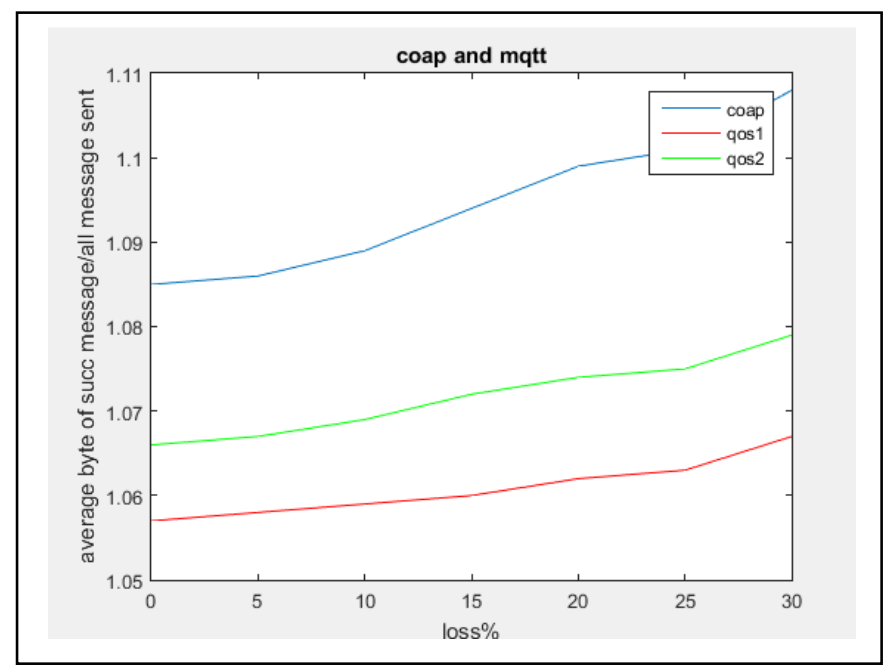

Fig. 11. IoT Average Byte "IAB".

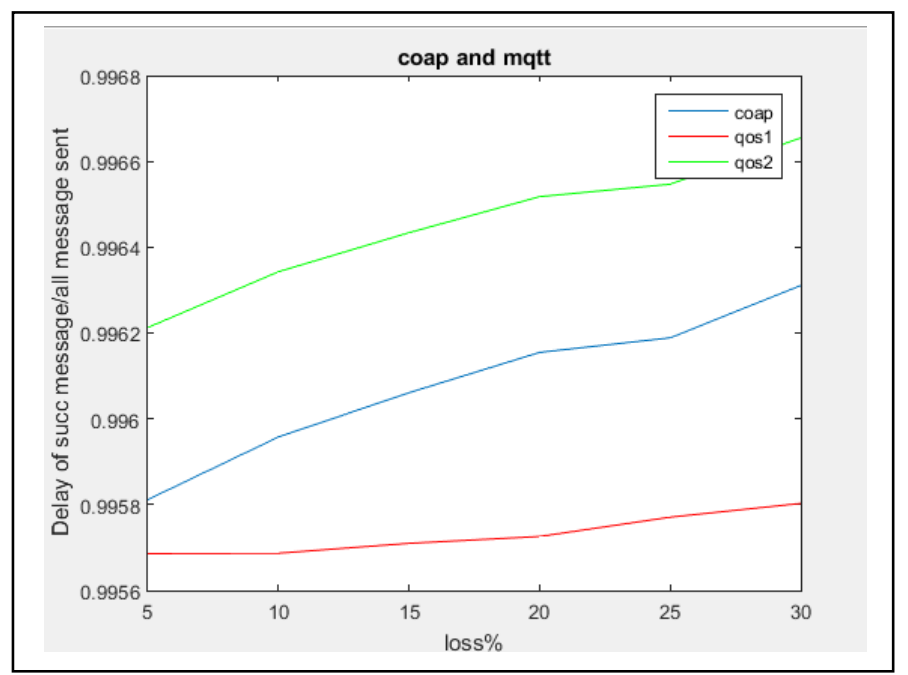

Fig. 12. IoT Delay Message "IDM". 


\section{CONCLUSION}

This paper presents design and implementation of e-health platform over IoT. A reliable e-health monitoring system over IoT has been designed and successfully implemented in this work based on MQTT and CoAP protocols. Whereas the proposed system is based on any type of internet access like WIFI, GPRS, 3G, so it is easily reconfigurable and can be extended to include more medical sensors and more parameters. The proposed e-health system is flexible enough to include such kind of modifications. Also, Comparing "MQTT \& CoAP" protocol to select the best IoT protocol for E-health applications is investigated in this paper. In particular, it defines real results depending on practical simulations to select the best IoT protocol for e-health applications. From the practical implementation of these protocols, MQTT protocol provided good results than CoAP protocol in the delay, the number of messages lost and the number of bytes used in messages. Thus decrease the energy used, and reduce the needed bandwidth. So from these practical and simulation results, MQTT message protocol more suitable to design and implementation of e-health platform over IoT.

\section{REFERENCES}

[1] B. Lee, "Healthcare Framework on the IoT open Platform," Service Model, Architecture, International Journal of Applied Engineering Research, vol. 9, pp. 29783-29792, 2014.

[2] M.Bhay, M. Pat el and Chin tan Bhatt," Internet of Things (IoT): In a Way of Smart World" () Springer Science, Singapore 2016.

[3] D. Uckelmann, M. Harrison, F. Michahelles "An Architectural Approach Towards the Future Internet of Things", Architecting the Internet of Things, Springer-Verlag Berlin Heidelberg 2011.

[4] U. Tandale, Dr. B. Momin and D. P. Seetharam " An Empirical Study of Application Layer Protocols for IoT" International Conference on Energy, Communication, Data Analytics and Soft Computing (ICECDS2017), IEEE, 2017.

[5] B. Farahani, F. Firouzi, V. Chang, M. Badaroglu ,N. Constant, K. Mankodiya " Towards fog-driven IoT E-Health: Promises and challenges of IoT in medicine and healthcare", Future Generation Computer Systems , 2018.

[6] J. Shah, S. Soni, F. Darji, S. Chandak, A. Shetty "Smart Hospital Using Iot "International Conference on Innovative and Advanced Technologies in Engineering, IOSR Journal of Engineering (IOSRJEN), Volume 9, PP 26-32. 2018.
[7] C. I. Saidu, A. S. Usman and P. Ogedebe, "Internet of Things: Impact on Economy", British Journal of Mathematics \& Computer Science, 2015.

[8] D. B. Ansari, A. Rehman, R. Mughal" Internet of Things (IoT) Protocols: A Brief Exploration of MQTT and CoAP", International Journal of Computer Applications (0975 - 8887) Volume 179 - No.27, 2018.

[9] Jorge Gómez, Byron Oviedo , Emilio Zhum, " Patient Monitoring System Based on Internet of Things", 7th International Conference on Ambient Systems, Networks and Technologies (ANT 2016), Published by Elsevier, 2016.

[10] R.N. Kirtana, Y.V. Lokeswari, " An IoT Based Remote HRV Monitoring System for Hypertensive Patients", IEEE International Conference on Computer, Communication, and Signal Processing (ICCCSP), IEEE, 2017.

[11] Antoine Jamin et al."An aggregation plateform for IoT-based healthcare: illustration for bioimpedancemetry, temperature and fatigue level monitoring", 2017.

[12] Kaleem Ullah et al.," Effective Ways to Use Internet of Things in the Field of Medical and Smart Health Care", International Conference on Intillegent System Engineering, IEEE, 2016.

[13] M. Hussein, M. Zorkany, N. Abdel Kader, "Design and Implementation of IoT Platform for Real Time Systems", International Conference on Advanced Machine Learning Technologies and Applications (Springer), Egypt, 2018.

[14] P. Thota, Y. Kim "Implementation and Comparison of M2M Protocols for Internet of Things" Intl Conf on Applied Computing and Information Technology,@ IEEE, 2016.

[15] K. Natarajan, B. Prasath, P. Kokila "Smart Health Care System Using Internet of Things" Journal of Network Communications and Emerging Technologies (JNCET) Volume 6, Issue 3, ISSN: 2395-5317 , 2016.

[16] A. Al-Fuqaha, M. Guizani, M. Mohammadi, M. Aledhari, Moussa Ayyash " Internet of Things: A Survey on Enabling Technologies, Protocols, and Applications", IEEE COMMUNICATION SURVEYS \& TUTORIALS, VOL. 17, NO. 4, 2015.

[17] T. Takpor and A. Atayero "Integrating Internet of Things and E-Health Solutions Students' Healthcare", Proceedings of the World Congress on Engineering 2015.

[18] S. Lee, H. Kim, D. Hong, Hongtaek Ju " Correlation Analysis of MQTT Loss and Delay According to QoS Level", IEEE ICOIN, 2013.

[19] B. Aziz, " A formal model and analysis of an IoT protocol" Ad Hoc Networks, Elsevier B.V. ,2016.

[20] H. C. Hwang ,J. Park, J. G. Shon," Design and Implementation of a Reliable Message Transmission System Based on MQTT Protocol in IoT" Wireless Pers Commun, Springer Science, New York ,2016. 